\title{
Über die Korngrenzendiffusion von flüssigem Wismut in Kupfer
}

\author{
Von Erich Scheil und Karl Eugen Schiessl \\ Ius dem Max-Planck-Institut für Metallforschung in Stuttgart \\ (Z. Naturforschg. 4 a, 524-526 [1949]; eingegangen am 20. März 1949) \\ Hrn. Prof. W. Gerlach zu seinem 60. Geburtstag überreicht
}

\begin{abstract}
Die Korngrenzendiffusion von flüssigem Wismut in Kupfer wurde mikroskopisch gemessen. Die Eindringtiefe ändert sich linear mit der Zeit. Der Logarithmus der Eindringgeschwindigkeit ist proportional der reziproken absoluten Temperatur. Bei ungeglühten Proben wurde im Bereich von 600 bis $900^{\circ}$ eine Änderung der Aktionskonstanten beobachtet, die nicht mit der Kornvergröberung zusammenhängt.

Oberhalb $700^{\circ}$ wird Kupferoxydul von Wismut reduziert, dadurch wird auch die bei tiefen Temperaturen auftretende Hemmung der Oberflächendiffusion beseitigt.

Bei tiefen Temperaturen wandert das Wismut vorwiegend längs Korngrenzkanten in das Kupfer hinein. Mit zunehmender Temperatur werden auch die Korngrenzflächen mit herangezogen, und dicht unter dem Schmelzpunkt werden alle Korngrenzflächen von der diffundierenden Schmelze bedeckt. Eine Ausnahme machen die Grenzen von Zwillingen.
\end{abstract}

$\mathrm{E}$ $\mathrm{s}$ sind drei Arten von Diffusionsvorgängen in einem kristallinen Körper zu unterscheiden:

1. Kristalldiffusion ${ }^{1}$ : Ein B-Atom ersetzt ein A-Atom auf seinem Gitterplatz oder tritt in eine Lücke ein. Dieser Vorgang erfolgt in einer homogenen Mischphase und kann nur bis zur Sättigungskonzentration der Kristallart gehen.

2. Korngrenzendiffusion²: Es erfolgt ein Stofftransport längs der Grenzflächen zweier bzw. lä̈ngs der Grenzkanten dreier Körner. Die Korngrenzensubstanz kann dabei der gleichen Kristallart wie die Muttersubstanz (homogene Korngrenzendiffusion) angehören, in der Regel wird sie jedoch einer anderen kristallinen oder flüssigen Phase angehören (heterogene Korngrenzendiffusion). Es sind noch weitere Fälle denkbar, nämlich daß die neue Phase sich längs einer Korngrenze zweier verschiedener Kristallarten ausbreitet, die man als heterogene Korngrenzendiffusion 2. Ordnung bezeichnen kann.

3. Oberflächendiffusion: Bei ihr breitet sich der diffundierende Stoff auf der Oberfläche der Probe aus. Als Beispiel sei die Diffusion von Quecksilber auf Zinn erwähnt ${ }^{3}$.

Während die Kristall- und die Oberflächendiffusion mehrfach untersucht worden ist, ist den Verff. aus der Literatur nur ein Fall einer Korn-

1 W. Seith, Diffusion in Metallen, J. Springer, Berlin 1939.

2 J. L a n g m u i r, Z. angew. Chem. 46, 719 [1933], zit. nach $\mathrm{Se}$ it h ${ }^{1}$.

3 W. Gerlach u. Schweitzer, S.-B. math.naturwiss. Aht. bayr. Akad. Wiss. 1930, 223. grenzendiffusion bekannt, nämlich die Diffusion des Thoriums in Wolfram. Ob es sich um einen homogenen oder einen heterogenen Fall handelt, ist noch ungewiß.

Uns sind drei Fälle einer heterogenen Korngrenzendiffusion bekannt, und zwar die Diffusion des fluissigen Kupfers in Eisen, des fliissigen

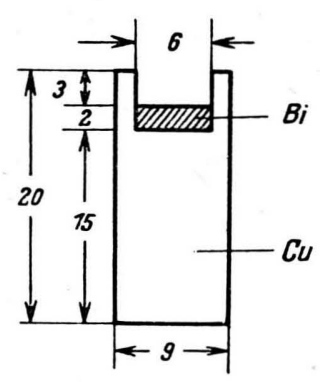

1b). 1. Probekörper.

Nickelsulfids in Nickel und des flüssigen Wismuts in Kupfer. Alle drei Erscheinungen ergeben eine starke Schädigung des Grundwerkstoffes. Über die Untersuchungen der Diffusion des Wismuts in Kupfer wird im folgenden berichtet.

Für die Versuche wurde eine Stange geglühten Walzkupfers (der Firma Kreidler in Stuttgart) benutzt. Die Probe von $9 \mathrm{~mm} \varnothing$ und einer je nach der Dauer des Versuchs verschiedenen Länge wurde an einer Stirnfläche um $5 \mathrm{~mm}$ ausgedreht (Abb. 1), sorgfältig entfettet und zur Füllung des ausgebohrten Teiles unter einer Wasserstoffatmosphäre kurze Zeit in flüssiges Wismut von etwa $400^{\circ}$ eingetaucht. Nach dem Erstarren wurden Mantel- und Bodenfläche durch schwaches 
Abdrehen vom Wismut befreit. Dann wurde sie in einem geregelten Ofen unter gereinigtem Wasserstoff geglüht. Nach dem Glühen wurde die Probe abgeschreckt, geschliffen und poliert. Oberhalb $700^{\circ}$ verdampfte das verwendete Wismut sehr rasch. Für das Glühen bei höherer Temperatur wurden deshalb die Proben in ein nachher zugeschweißtes Eisenrohr eingebracht.

Da es sich um eine heterogene Korngrenzendiffusion handelt, so kann die Eindringtiefe mikroskopisch gemessen werden. Die bläulichen Wismutadern sind im ungeätzten, sorgfältig polierten Schliff gut von der Kupfergrundmasse zu unterscheiden.

Abb. 2 zeigt die isotherme Abhängigkeit der Eindringtiefe $E$ von der Glühzeit für einige Tem-

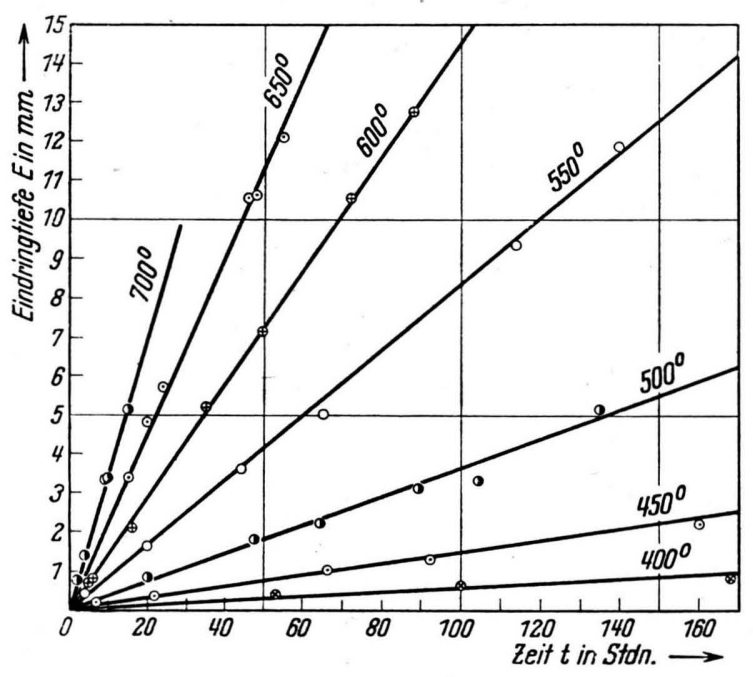

Abb. 2. Isothermen der Eindringtiefe des Wismuts in Kupfer.

peraturen. Die Eindringtiefe nimmt proportional mit der Zeit zu, d. h. das Eindringen wird durch die Dicke der bereits durchdrungenen Schicht nicht gehemmt. In Abb. 3 ist der Logarithmus der Proportionalitätskonstanten $a=d E / d t$ in Abhängigkeit von der reziproken abs. Temperatur aufgetragen. Die ausgezogene Kurve gibt die Messungen an Proben wieder, die vor dem Ausbohren $1 / 2$ Stde. bei $1000^{\circ}$ geglüht worden waren. Die Konstante $a$ gehorcht der Gleichung

$$
\ln a=\ln A-\frac{Q}{R T} .
$$

Für die Aktivierungsenergie $Q$ erhält man den Betrag von $20000 \mathrm{cal} /$ Grammatom. $A$ bezeichnet man als die Aktionskonstante.
Die Konstanz der Aktivierungsenergie ist sehr merkwürdig. Mit zunehmender Temperatur nimmt nämlich der Kupfergehalt der Schmelze zu, wie Tab. 1 zeigt. Diese Konzentrationsänderung ist ohne Einfluß auf die Aktivierungsenergie und die Aktionskonstante.

Im Anlieferungszustand war das Kupfer geglüht, wie aus dem Gefüge zu sehen war. Glühtemperatur und Glühdauer waren aber unbekannt.

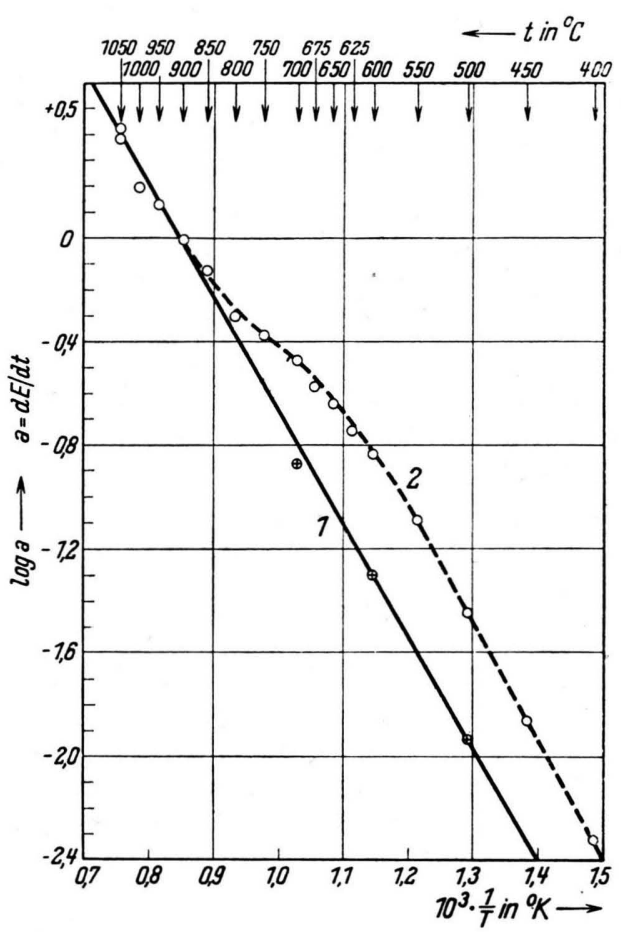

Abb. 3. Temperaturabhängigkeit der EindringtiefeKonstanten.

Bei diesen Proben war die Eindringgeschwindigkeit $a$ wesentlich größer als bei den bei $1000^{\circ}$ vorgeglühten Proben. Die gestrichelte Kurve in Abb. 3 gibt die Meßergebnisse an diesen Proben wieder. Sie verläuft bei tiefen Temperaturen

\begin{tabular}{|c|c|}
\hline $\begin{array}{c}\text { Temperatur } \\
{ }^{\circ} \mathrm{C}\end{array}$ & $\begin{array}{c}\text { Cu-Gehalt } \\
\text { in } \%\end{array}$ \\
\hline 400 & 3,6 \\
500 & 7 \\
600 & 12 \\
700 & 21,5 \\
800 & 41 \\
900 & 73 \\
1000 & 91,5 \\
\hline
\end{tabular}

Tab. 1. Kupfergehalt der gesättigten Wismutschmelze. 
parallel der ausgezogenen. Von etwa $600^{\circ}$ weicht sie von der Geraden ab und mündet bei etwa $900^{\circ}$ in die ausgezogene Kurve ein. Die Aktionskonstante $A$ in Gl. (1) ändert sich also zwischen 600 und $900^{\circ}$ bei der im Anlieferungszustand vorliegenden Kupferprobe, während die Aktivierungsenergie $Q$ unverändert bleibt.

Es lag nahe, die Kornvergrößerung als Ursache der Änderung anzunehmen. Zur Prüfung wurde die Korngröße der dem Eindringen des Wismut ausgesetzten Proben in den noch wismutfreien

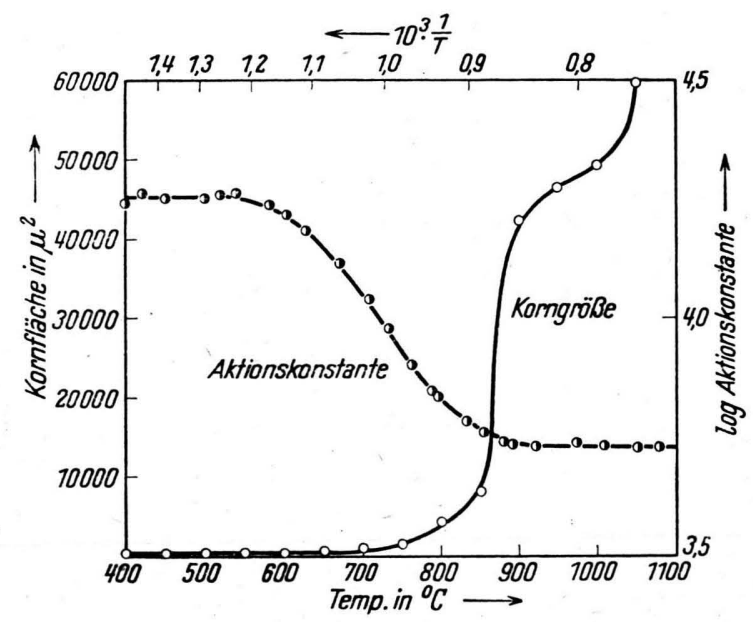

Abb. 4. Änderung der Korngrößen und der Aktionskonstanten mit der Glühtemperatur.

Gebieten gemessen. Abb. 4 zeigt die Änderung der Korngröße und der Aktionskonstanten $A$ mit der Temperatur. Offenbar besteht zwischen den beiden Änderungen kein einfacher Zusammenhang.

Man sollte erwarten, daß Fälle mit Korngrenzendiffusion auch Oberflächendiffusion zeigen. Die Oberflächendiffusion sollte sogar wesentlich rascher erfolgen. Das trifft auch, abgesehen von der Diffusion des Thoriums in Wolfram, bei den beiden weiteren Beispielen flüssiges Kupfer in Eisen und flüssiges Nickelsulfid in Nickel zu, nicht aber bei dem hier untersuchten Beispiel, wenigstens nicht bis etwa $700^{\circ}$.

Die Ausnahme ist nur scheinbar. Ab $700^{\circ}$ findet man auch beim Wismut Oberflächendiffusion, die bei tieferen Temperaturen durch eine Oxydhaut auf dem Kupfer gehemmt wird. Die Beseitigung der Hemmung erkennt man auch daran, daß oberhalb $700^{\circ}$ das Wismut mit dem Kupferoxydul im
Innern der Körner reagiert. Dabei verschwinden die im polarisierten Licht rubinroten Kupferoxyduleinschlüsse, und an ihre Stelle tritt eine neue Kristallart, wahrscheinlich Wismutoxyd oder ein ternäres Oxyd.

Mit steigender Temperatur ändert sich offenbar auch der Eindringweg des Wismuts in Kupfer.

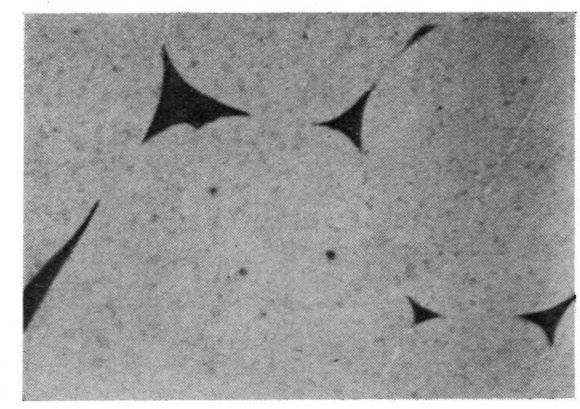

Abb. 5. Eindringen des Wismuts bei $600^{\circ}$ längs der Korngrenzkanten.

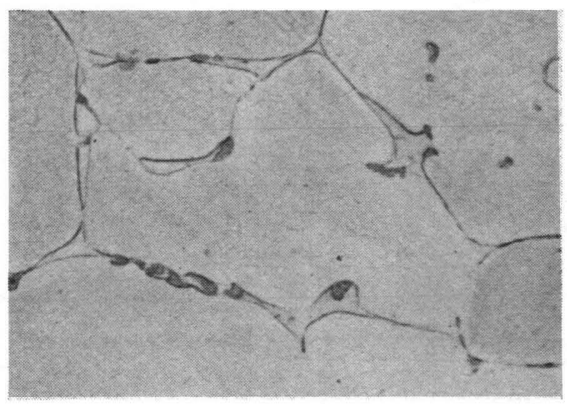

Abb. 6. Eindringen des Wismuts bei $850^{\circ}$ längs der Korngrenzflächen.

Bei tiefen Temperaturen wandert das Wismut vorwiegend längs der Korngrenzkanten in das Kupfer ein (Abb. 5). Korngrenzflächen werden selten belegt. Es ist nicht einmal sicher, ob die im Schliffbild als Korngrenzflächen angesprochenen Gebilde nicht auch in Wirklichkeit Ausläufer einer im Schliff nicht sichtbaren Korngrenzkante sind. Bei $850^{\circ}$ (Abb. 6) sind die Korngrenzen schon so häufig belegt, daß es sich hier bereits vorwiegend um Korngrenzflächen handeln muß. Schließlich sind bei etwa $1000^{\circ}$ praktisch alle Korngrenzen belegt. Eine Ausnahme machen lediglich die Zwillingsgrenzen, auf denen in keinem Fall Wismutfilme beobachtet worden sind. 